\title{
Transformación de espacios educativos abandonados con estrategias de organización comunitaria
}

Transformation of educational spaces abandoned with community organization strategies

Angie Serrano

profangieserrano2020@gmail.com

Código ORCID: 0000-0003-3124-983X

Unidad Educativa Nacional Dr. Elías Rodríguez, Caracas, Venezuela

Recibido abril 2019 | Arbitrado mayo 2019 | Publicado julio 2019

\section{Resumen}

Palabras clave:

Organización

comunitaria; espacios

educativos; rol del

adulto, participación
El objetivo de este trabajo fue proponer estrategias de organización comunitaria dirigidas a los adultos que hacen vida en una institución educativa para la trasformación de los espacios abandonados en unos de aprendizajes educativos. La metodología fue de tipo investigación documental con un diseño bibliográfico conjuntamente con los aportes una de campo de nivel descriptivo. La institución seleccionada fue la Unidad Educativa Dr. Elías Rodríguez, por ser un espacio donde había desidia y poca participación de los actores que hacen vida en dicho plantel. Lo que se buscó con las estrategias que se diseñaron fue crear un espacio educativo en el que los que laboran allí, personal administrativo, obrero, directivo y docente, así como sus estudiantes, sean quienes contribuyan con el mantenimiento de su infraestructura, de tal manera que sea un espacio agradable, armónico y limpio que propicie el aprendizaje y que brinde bienestar a toda la comunidad.

\section{Abstract}

\section{Keywords:}

community

organization;

educational spaces;

role of the adult; participation
The purpose of this work was to propose community organization strategies aimed at adults who make life in an educational institution for the transformation of abandoned spaces into educational learning spaces. The methodology was of documentary research type with a bibliographic design together with the contributions of a descriptive level field. The selected institution was the Dr. Elías Rodríguez Educational Unit, as it was a space where there was laziness and little participation from the actors who make life on said campus. What was sought with the strategies that were designed was to create an educational space in which those who work there, administrative staff, workers, managers and teachers, as well as their students, are those who contribute to the maintenance of their infrastructure, in such a way Make it a pleasant, harmonious and clean space that fosters learning and that provides well-being to the entire community. 


\section{INTRODUCCIÓN}

El ser humano, como ser social, va construyendo su vida dentro de los diversos grupos sociales donde le corresponde vivir; por ello, las organizaciones hacen parte de su cotidianidad como espacios de crecimiento. Se entiende a las organizaciones como sistemas de relaciones de personas y procesos en función de la producción de bienes 0 servicios que requieren las sociedades para su existencia. Así, cada organización existe en función de otras, en una red que forma la sociedad como un todo histórico (Universidad Nacional Experimental de las Fuerzas Armada [UNEFA], 2011).

Una de esas organizaciones es la llamada organización comunitaria cuya importancia radica en dar a conocer los valores humanos y talentos individuales para resolver de forma efectiva los problemas sociales, económicos y políticos de la comunidad. Mujeres y hombres como seres sociales, necesitan de la organización con sus semejantes para comunicarse, socializarse, desarrollarse integralmente y buscar condiciones más justas para la sociedad en la cual viven (Vaagt y Ruiz, 2008).

Las organizaciones no están solas, ni actúan solas en la sociedad. Generalmente, hay otras organizaciones, algunas de ellas con objetivos similares, otras con objetivos diferentes y algunas hasta con objetivos opuestos, por ello no se puede actuar en la sociedad, sin considerar este factor. Esto lleva a establecer relaciones con otras organizaciones, tal es el caso de la escuela como espacio educativo.

La escuela, como institución social, está obligada a establecer una interacción directa con el entorno en el cual se encuentra. Es necesario que esta cumpla plenamente con su propósito socializador para que exista correspondencia con la realidad cultural de los estudiantes, del entorno familiar y del ámbito social. Para Meza (2011), la sociedad actual debe dejar de concebir a la escuela como un espacio donde los niños y jóvenes acuden con el propósito de aprender unas materias. La institución debe transformarse en un complejo educacional cuyo objetivo sea responder, de la mejor manera, a las demandas y necesidades de la comunidad a la que presta sus servicios.

El currículo se considera el eje central que contiene todos los elementos de transformación para generar los cambios que demanda la sociedad venezolana. Las implicaciones epistemológicas en el currículo están dirigidas a la formación de un individuo crítico con aptitudes y habilidades, en términos del ser, saber, saber hacer y convivir con la realidad social inmediata. De allí que lo social y comunitario sean partes esenciales del proyecto. En el documento Bases Conceptuales y Operativas de las Escuelas Bolivarianas (Ministerio de Educación, Cultura y Deportes, 2001) se destaca la escuela como espacio comunitario, esta debe propiciar una apertura al trabajo desde lo comunitario, más allá de los límites del aula.

En la escuela bolivariana, la instrucción está centrada en el sujeto y contextualizada con el acervo histórico y cultural de la comunidad a la que pertenece el centro educativo. Esta escuela ha sido diseñada para integrarse a la comunidad, a través de la participación de los miembros en la construcción del proceso educativo. Es así 
como estudiantes, docentes y comunidad representan los entes activos de una serie de experiencias que requieren vincularse contribuyendo con la ruptura de viejos paradigmas, para asumir el reto en la adquisición de herramientas que fortalezcan un conocimiento al servicio de la sociedad y en contribución a una mejor calidad de vida de esta (Rodríguez, 2014).

Uno de los instrumentos propuestos para lograr este fin es el Proyecto Educativo Integral Comunitario (PEIC). Este se fundamenta en una concepción holística y específica del hecho educativo, donde se analizan las aspiraciones sentidas por el colectivo que conforma el plantel y su entorno, para luego generar alternativas que permitan el mejoramiento de la calidad de la educación como un hecho de significación social.

El proceso educativo, tal como lo establece la Constitución de la República Bolivariana de Venezuela (1999), está estrechamente vinculado al trabajo a fin de armonizar la educación con las actividades productivas propias del desarrollo social local, regional y nacional, a través de la orientación de niños, niñas, adolescentes y jóvenes; formándolos en, por y para el trabajo creador y productivo, con una visión dignificadora de lo humano que permita satisfacer necesidades básicas y avanzar hacia una mejor calidad de vida.

Tal como lo expresa González (2007), “la participación ciudadana es el proceso mediante el cual los ciudadanos en forma directa 0 mediante sus expresiones asociativas, inciden en ciertos procesos gubernamentales definitorios de políticas públicas" (p. 56). Por lo que la participación de ciudadanos, individuos, comunidades y sectores sociales organizados tienen la oportunidad de intervenir de distintas maneras en la resolución de determinados asuntos de interés común.

Desde esta perspectiva, los programas de educación deben ser impartidos de manera tal que se logren propiciar espacios de aprendizajes que vinculen a la escuela con la comunidad, orientando una gestión educativa destinada al servicio comunitario del colectivo. La colectividad no solo tiene algo que decir sobre lo que afecta a su vida como persona, miembros de una familia $y$ comunidad, sino que también deben ser informados, consultados y escuchados en cuanto a todos los aspectos.

Así la escuela debe demostrar que hay una integración, vinculación, y sentido de participación con la comunidad porque la innovación pedagógica, las nuevas técnicas de aprendizaje y la apertura creciente de las instituciones educativas a los problemas fuera del ámbito escolar, requieren de un mayor compromiso y acción por parte de todos los miembros de la comunidad educativa, a los fines de que realmente la integración del contexto al entorno educativo sea real y factible.

En este sentido, se hace necesario revisar qué está ocurriendo en las escuelas para constatar si, a través del Proyecto Educativo Integral Comunitario (PEIC), se están logrando estas políticas de Estado, porque pareciera que no se está evidenciando la correspondencia entre el desarrollo social con los procesos educativos desde los diferentes ámbitos de acción de la educación: formal, no formal e Informal, para contribuir con una formación ciudadana refundada desde un nuevo enfoque humanista constructivista. Para ello 
se requiere que desde los diferentes escenarios de vida de la sociedad: políticos, educativos y sociales se fundamente un modelo productivo que articule la acción de las entidades organizadas para transformarlos en agentes dinamizadores dentro de sus contextos.

Actualmente, el docente como mediador se desenvuelve muchas veces aislado de la realidad social local. Su actividad ha estado centrada fundamentalmente en el trabajo pedagógico del aula cuando lo que se requiere es un docente con una participación más activa en función de la transformación de su realidad y en la construcción de un entorno social más armónico. Por su parte, la comunidad no se involucra en la participación de actividades conjuntas con las instituciones educativas y que consideren importantes para el bienestar individual y colectivo. No existe una cultura de participación ciudadana que busque la atención de diferentes problemáticas sociales desde la escuela.

En el Proyecto Educativo Integral Comunitario elaborado por la Unidad Educativa Nacional "Dr. Elías Rodríguez", durante el año escolar 2013 -2014 y 20142015 se reportó entre las debilidades: a) apatía por parte de algunos docentes para integrarse a las actividades desarrolladas en las instituciones educativas en favor de la comunidad, b) débil relación entre la comunidad y la escuela, c) falta de preocupación e integración de los representantes en las actividades de sus hijos, rivalidades entre algunos de los sectores de la parroquia, y e) poca conciencia por parte de la comunidad en valorar y mantener las obras realizadas en la parroquia tales como limpieza de alcantarillas, alumbrado eléctrico, entre otros. Situación que se viene repitiendo en los años escolares sucesivos.

Por otro lado, se ha venido observando cómo a partir del estado de abandono en que se encuentran los diferentes espacios de este plantel ubicado en la Parroquia San Agustín del Sur se observa: desidia, desinterés, poca participación de los actores que hacen vida allí, lo que afecta seriamente la calidad educativa que se le brinda al estudiante y donde los espacios y servicios tales como: canchas, infraestructura física, baños, biblioteca, sistema eléctrico, agua servidas, inoperancia de los equipos del Centro Bolivariano de Informática y Telemática (CBIT), Sistema de Alimentación Escolar (SAE) y aulas, entre otros, se encuentran abandonados. Aunado a esto, los actores principales obvian tal situación.

Por lo antes expuesto, la presente investigación se planteó como objetivo general y específicos los siguientes (Serrano, 2018):

\section{Objetivo general}

Proponer estrategias de organización comunitaria dirigidas a los adultos que hacen vida en la Unidad Educativa Nacional Dr. Elías Rodríguez para la trasformación de los espacios abandonados en espacios amigables de aprendizajes educativos

\section{Objetivos específicos}

1. Analizar los elementos teórico-filosóficos y socioculturales contemplados en el diseño curricular del sistema educativo venezolano, que propicien la organización comunitaria para la transformación de los espacios educativos. 
2. Describir la actitud de los actores que hacen vida en la Unidad Educativa Nacional Dr. Elías Rodríguez, en relación con su participación- en los procesos de gestión institucional.

3. Diseñar estrategias que orienten la organización comunitaria en la Unidad Educativa Nacional Dr. Elías Rodríguez, en relación con su participación en los procesos de gestión institucional.

\section{La organización comunitaria}

Organización es una palabra que deriva del latín "organón" y significa órgano o elemento de un sistema. Por tanto, es un conjunto de cargos cuyas reglas y normas de comportamiento deben sujetarse a todos sus miembros. Una organización comunitaria, por tanto, es aquella organización con personalidad jurídica y sin fines de lucro, que tiene por objeto representar y promover valores e intereses específicos de la comunidad (Guánchez, 2014).

Las organizaciones son formas de participación que existen o pueden existir en las comunidades y que agrupan a un conjunto de ciudadanos y ciudadanas en base a objetivos e intereses comunes. El fin de la organización comunitaria es propiciar la unidad dentro de la diversidad, para abordar, intervenir y participar en la transformación de su entorno social; permitiendo elevar la calidad de vida y alcanzar el bienestar colectivo. En otras palabras, la organización comunitaria es en sí misma, una acción transformadora y liberadora de la sociedad. Dentro de la organización comunitaria, deberían prevalecer algunos valores primordiales, tales como: solidaridad, flexibilidad, dinámicas pro activas, compartidas y con capacidad de adaptación, entre otros.

La organización comunitaria es importante porque es ahí donde se dan a conocer los valores humanos y talentos individuales para resolver de forma efectiva los problemas sociales, económicos y políticos de la comunidad. Los individuos como seres sociales necesitan de la organización para comunicarse y desarrollarse integralmente.

En Venezuela este proceso es ejercido a través de una figura llamada los consejos comunales, que son entes de participación ciudadana orientados a solventar las necesidades de la comunidad que los forma e integra, en general este proceso fortalece el poder popular y el desarrollo y defensa de una nación. La Ley Orgánica de los Consejos Comunales (2009) define a las organizaciones comunales como "organizaciones que existen o pueden existir en el seno de las comunidades y agrupan un conjunto de personas con base a objetivos e intereses comunes, para desarrollar actividades propias en el área que les ocupa" (p. 2).

Por su parte, la comunidad se constituye como un espacio local que cumple la función de ser el motor estratégico para la transformación de la realidad social, donde los sujetos sociales participan de manera activa en la construcción de realidades colectivas contribuyendo así, a que los actores institucionales puedan ser enriquecidos y controlados por el aporte de sus miembros, de allí que se defina “... como un espacio básico de convivencia social, en el cual convergen un conjunto de grupos y de personas que interactúan entre sí, en el marco de reglas y valores 
establecidos" (Gómez y Millán, 2002, p. 108).

Frente a todas estas ventajas, es necesaria la incorporación de las escuelas a las organizaciones comunitarias. De allí que sea importante reconocer el rol histórico de la escuela como la institución destinada a la producción, apropiación y distribución de los bienes culturales en una comunidad determinada (Ministerio de Educación, Ciencia y tecnología, s/f). El trabajar conjuntamente en la elaboración de proyectos educativos entre la escuela y otras organizaciones o instituciones de la comunidad, posibilita la pronta visualización de los niños en situación de vulnerabilidad educativa y aquellos que se encuentran por fuera de la escuela.

El Ministerio de Educación, Cultura y Deportes (2009) contempla que la escuela será un espacio para la vida comunitaria y la comunidad un recurso para el proceso formativo del alumnado. Siempre y cuando la escuela no pierda el foco para lo que fue creada y la comunidad entienda cuáles son sus limitaciones para influir en el accionar académico. "El mejor ejemplo lo tenemos en el funcionamiento de las escuelas de Fe y Alegría en Venezuela" ( $p$. 5).

El Ministerio del Poder Popular para la Educación en Venezuela en el año 2012 promulgó la Resolución 058, la cual tiene como propósito crear el Consejo Educativo. Con la creación de este se pretende desarrollar soluciones a los problemas que se presenten en cada institución educativa, yendo incluso más allá de los muros del recinto escolar. De allí que la organización de la comunidad educativa deba establecerse a través del Consejo Educativo.

El Consejo Educativo está conformado por padres, madres, representantes, responsables, estudiantes, docentes, trabajadores administrativos y obreros. También podrán formar parte personas naturales y jurídicas, voceros de las diferentes organizaciones comunitarias vinculadas con las instituciones educativas. Se organizan en los diferentes comités: Contraloría Social, académico, seguridad y defensa integral, comunicación e información, ambiente y salud integral, hábitat escolar, salud, madres, padres, representantes y responsables, deporte y actividad física y estudiantes.

Todos los responsables y corresponsables de la gestión escolar participan activa y directamente y resuelven los asuntos de interés de la comunidad educativa a través de sus vocerías en los comités. La toma de decisiones se realiza en colectivo, mediante una puesta en común y promueve espacios de articulación con la comunidad.

\section{Proyecto Educativo Integral Comunitario (PEIC)}

El Proyecto Educativo Nacional (Ministerio de Educación, Cultura y Deportes, 1999) plantea la necesidad de formar desde las instituciones educativas una cultura de participación ciudadana que permita la atención de diferentes problemáticas del entorno local y regional. Así mismo, en el documento Bases Conceptuales y Operativas de las Escuelas Bolivarianas (Ministerio de Educación, Cultura y Deportes, 1999) se destaca la escuela como espacio comunitario; esta debe propiciar una apertura al trabajo 
desde lo comunitario, más allá de los límites del aula.

Uno de los instrumentos propuestos para lograr este fin es el Proyecto Educativo Integral Comunitario (PEIC), el cual se fundamenta en una concepción holística y específica del hecho educativo, donde se analizan las aspiraciones sentidas por el colectivo que conforma el plantel y su entorno, para luego generar alternativas que permitan el mejoramiento de la calidad de la educación como un hecho de significación social.

EI PEIC se desarrolla en diversas etapas. Son estas: diagnóstico, planificación y evaluación. La etapa de diagnóstico busca explorar de forma concreta la situación de la escuela y su entorno (Aguilar y Ander-Egg, 2001). La etapa de planificación se definen los objetivos generales y específicos, se concretan las acciones para abordar los problemas encontrados y se asignan 0 distribuyen las responsabilidades. El plan de acción tiene como propósito fundamental definir y organizar en grupo las actividades y acciones para alcanzar los objetivos propuestos. La etapa de evaluación permite valorar lo que se ha hecho y darse cuenta de la efectividad 0 no de las acciones desarrolladas.

\section{MÉTODO}

Por su contenido y forma, este estudio estuvo enmarcado en una investigación documental, ya que toda la información se obtuvo a través de materiales impresos y electrónicos seleccionando temas relacionados con el tópico de la naturaleza del objetivo investigado. La autora de este estudio llevó a cabo un proceso de indagación bibliográfica a fin de recolectar información y analizarla para dar respuestas a los objetivos previamente planteados en este estudio.

En cuanto al diseño de la investigación, está basada en un diseño bibliográfico porque se hizo una revisión de referencias de manera exhaustiva, agotando todo lo relacionado con el tema en estudio. Para Bisquerra (2004) "la investigación bibliográfica es aquella donde se explora qué se ha escrito en la comunidad científica sobre un determinado tema" (p. 78).

De acuerdo con las características de la presente investigación, se enmarcó en un nivel descriptivo que, según Hernández, Fernández y Baptista (2010), buscan expresar la manera cómo se lleva a cabo un fenómeno, a través de la descripción de dichas situaciones o eventos.

Las técnicas empleadas fueron el análisis de contenido y el fichaje. El análisis de contenido constituye una técnica de estudio para observar, razonar, comparar, cotejar y determinar una información consultada en algunos documentos previamente revisados y que son tomados como referencias en una investigación (Sánchez, 1998).

El fichaje es el proceso de recopilación y extracción de datos importantes de fuentes bibliográficas como libros, revistas, periódicos e internet para el desarrollo de una investigación (Hernández, Fernández y Baptista, 2010).

En cuanto al instrumento utilizado fue la ficha de trabajo o ficha de contenido específico, en las cuales se registran los datos extraídos de los documentos objeto de estudio, así como también las anotaciones personales producto de las reflexiones del investigador en relación al tema que investiga (Mengo, 2009). 
El procedimiento seguido se dividió en tres momentos:

\section{Etapa I. Análisis de los elementos teórico-filosóficos y socioculturales. En} esta fase inicial, se procedió a analizar los elementos teórico-filosóficos y socioculturales contemplados en el diseño curricular del sistema educativo venezolano. Para ello, se realizó una revisión y análisis al Currículo Básico Nacional y otros documentos inherentes al tema para sistematizar tales elementos que propician la organización comunitaria para la transformación de los espacios educativos.

\section{Etapa II. Participación en los procesos} de gestión. Una vez seleccionado y delimitado el tema, se procedió a determinar la problemática que se iba a abordar en torno la actitud de los actores que hacen vida en la Unidad Educativa Nacional Dr. Elías Rodríguez, en relación con su participación en los procesos de gestión institucional.

Etapa III. Diseño de estrategias. En esta etapa, la autora se diseñó estrategias para orientar la organización comunitaria en la Unidad Educativa Nacional Dr. Elías Rodríguez, en relación con su participación en los procesos de gestión institucional.

RESULTADOS

\section{Etapa I. análisis de los elementos teórico-filosóficos y socioculturales}

El Currículo Nacional Bolivariano: Diseño Curricular del Sistema Educativo Bolivariano (Ministerio del Poder Popular para la Educación, 2007) dicta las bases históricas, pedagógicas, filosóficas, sociales, culturales, psicológicas, políticas, metodológicas, científicas y humanistas de la formación de los niños, niñas, jóvenes, adultos y adultas, en los subsistemas que le competen al Ministerio del Poder Popular para la Educación. Plantea que los principios, características, perfiles, orientaciones, propósitos, componentes y metodología de aprendizaje de este currículo están fundamentados en las ideas y praxis libertadoras, filosóficas, pedagógicas, políticas, sociales, culturales de ilustres venezolanos como Simón Rodríguez, Francisco de Miranda, Simón Bolívar, Ezequiel Zamora, Luis Beltrán Prieto Figueroa, Belén Sanjuán y de eminentes pensadores, pedagogos de América Latina y del mundo (p. 2).

En el contexto venezolano, Luis Beltrán Prieto Figueroa es una de las figuras contemporáneas que nutre esta propuesta curricular. Prieto Figueroa (2006) consideraba la educación como un fenómeno colectivo, regido por normas establecidas por un grupo social, expresado como la totalidad; razón por la cual, el Estado debe determinar los medios para satisfacerla. De este planteamiento, se desprende la idea de que la educación debe formar integralmente al nuevo republicano y la nueva republicana, haciendo énfasis en el desarrollo humanístico y acorde con un contexto social específico.

La educadora venezolana Belén Sanjuán, firme seguidora de los postulados educativos de Simón Rodríguez y del maestro Luis Beltrán Prieto Figueroa, afirma que la educación debe ser integral, preparar 
para la vida, en la medida en que se concibe como un método pedagógico que permita el desarrollo de la totalidad de la personalidad de los estudiantes y los maestros.

Esta concepción de la educación alimenta una pedagogía latinoamericana, de la que también forma parte el ideario educativo de José Martí, quien aborda la educación como un acto pedagógico permanente, que permite el desarrollo integral del nuevo republicano.

Freire (2004) concibe a la educación como proceso que sirve para que los estudiantes y los maestros aprendan a leer la realidad para escribir su historia, lo cual supone comprender críticamente su mundo y actuar para transformarlo.

Las ideas expuestas por estos pensadores, definen una concepción de la educación según la cual estudiantes son protagonistas de múltiples interacciones sociales, participantes activos en los procesos de evaluación y organización de las experiencias de aprendizaje, caracterizándose por ser curiosos, creadores, cooperativos, transformadores, problematizadores de saberes, humanistas, experimentadores y por estar en constante búsqueda de soluciones originales de los problemas de su entorno local, regional y nacional.

Además, permite caracterizar a los maestros como activadores, mediadores de los saberes, el sentir, el hacer social y cultural y el proceso de apropiación de los aprendizajes por parte de los estudiantes. Al mismo tiempo que los identifica como propiciadores de experiencias de aprendizaje, en una unidad dialéctica entre teoría y práctica; promotores del diálogo, la discusión y el autoaprendizaje, desde una relación comunicativa centrada en un ambiente de afectividad y tolerancia; siendo capaces de despertar en el estudiante el deseo de ser útil a la sociedad, de ser merecedor de una vida digna, feliz y alegre, en relación a su contexto social y cultural. Debe ser ético, comunicativo, accesible, congruente en el pensar, actuar y sentir, con amplio patrimonio cultural, sensible al lenguaje y a la cultura popular, y propiciar el aprender a reflexionar.

\section{Etapa II. Participación en los procesos de gestión}

Los actores hacen referencia a todas aquellas personas que estén involucradas en la actividad dentro de una organización, ya sea en forma individual o colectiva, quienes a su vez forman alianzas para conseguir los objetivos del grupo (Crozier y Friedberg, 1990). Estos actores son actores sociales con protagonismo sobre la organización.

Para estos autores, el actor se convierte en agente, en promotor de luchas y negociaciones, que maneja sus posibilidades para ejercer poder y lograr sus fines individuales y de grupo, plantean que una persona implicada en una situación no es pasiva jamás; esta persona es un actor que tiene una estrategia propia y trata de mantener su zona de poder (Crozier y Friedberg, 1990, p. 25).

En cuanto a la participación, es una acción social que consiste en intervenir activamente en las decisiones y acciones relacionadas con la planificación, la actuación y la evaluación de la actividad que se desarrolla en el centro y en el aula. Se refiere a actos deliberados y conscientes 
que nacen del derecho consustancial de los ciudadanos a construir libre y responsablemente, como protagonistas, la realidad que viven (Coronado 2010, p. 4).

\section{Etapa III. Diseño de estrategias}

En esta tercera y última etapa, se diseñaron algunas estrategias para orientar la organización comunitaria en la Unidad Educativa Nacional Dr. Elías Rodríguez, en relación con su participación en los procesos de gestión institucional. Se diseñaron seis estrategias:

1. De liderazgo social, dirigida al personal directivo (ver Cuadro 1).
2. De gestión de las políticas públicas educativas, destinada al Consejo Educativo (ver Cuadro 2).

3. De formación y preparación, dirigida a los docentes en servicio (ver Cuadro $3)$.

4. De participación social, destinada a los padres y representantes (ver Cuadro 4).

5. De participación estudiantil, dirigida a los estudiantes (ver Cuadro 5).

6. De servicios de apoyo a la institución, está dirigida al personal administrativo y obrero (ver Cuadro 6). 
Cuadro 1. Estrategia de liderazgo social

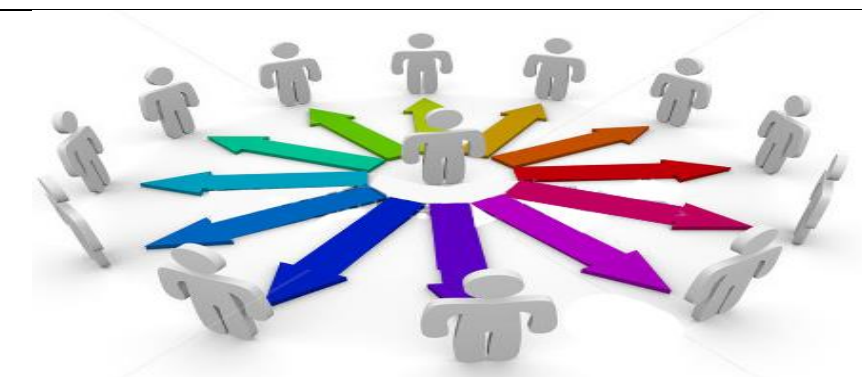

Actividades

1. Motivar a su personal en general para hacerlos sentir útiles y necesarios en la comunidad.

2. Promover la interrelación escuela- comunidad como líder social, en beneficio de la atención de los estudiantes.

3. Mantener una comunicación directa con el docente para conocer sus inquietudes, preocupaciones y necesidades en el ámbito escolar. El conocimiento personal le sirve para crear un grupo cooperativo y motivarlo a sacar adelante los proyectos del colegio. Debe incluir a los docentes en el proceso de toma de decisiones (participación consultiva).

\section{Dirigida: Al personal directivo}

Objetivo: Liderizar la búsqueda de soluciones a los problemas críticos del plantel.

Hacer un sondeo que cuántos representantes o docentes de la escuela viven en la comunidad, e incluso si forman parte de nuestra organización o de alguna otra expresión comunitaria. Es pertinente hacer un directorio con sus datos, para convocarles especialmente, sería estupendo que ellos recomendasen iniciativas o sirviesen de enlace permanente. Hay que procurar armonía, sintonía y articulación entre la escuela y la comunidad. 
4. Convocar y dirigir reuniones con el personal en general y con la comunidad a fin de plantear y analizar temas relacionados con las problemáticas que vive la escuela con el fin de buscar soluciones entre todos.

5. Conformar el Consejo Educativo a fin de liderizar el diseño de estrategias a través del P.E.I.C.

6. Liderizar una cultura de participación ciudadana que permita la atención de diferentes problemáticas de la institución
- Propiciar un primer encuentro entre todos los entes que hacen vida en la escuela.

- Hacer seguimiento a la convocatoria para que la misma sea positiva y masiva.

- Consolidar el primer encuentro de integración escuela-comunidad.

Extraer proposiciones de los diversos sectores que asistan al encuentro.

Ejecutar la actividad conocida como "evaluación de necesidades" donde se concientiza a todos sobre la gama completa de problemas y sus diversas causas, y se determina cuáles problemas se pueden resolver a corto y cuáles a largo plazo.

Caminata de observación: ¿Cómo está mi comunidad? Durante las caminatas de observación la gente observa de cerca a su comunidad. Trata de descubrir las causas posibles de los problemas de salud, por ejemplo fuentes inseguras de agua, el estado de los sanitarios, etc. Cuando el grupo termina la caminata, los participantes intercambian opiniones sobre su conocimiento de los problemas y así pueden luego encontrar juntos las soluciones correspondientes.

Libreta de contactos: Prepara una libreta con el nombre (en orden alfabético) de los recursos de la comunidad que ya hayan sido identificados e intégralos en actividades del centro o escuela. Clasifícalos de acuerdo al trabajo que desempeñan, a los servicios que ofrecen o los lugares de la comunidad. Incluye el teléfono dónde localizarlos.

Identificar organizaciones, programas o servicios: Estos programas pueden ser de las áreas de salud, cultura, servicios sociales y recreativos, que estén localizados en la comunidad y que trabajan con familias. Los mismos son necesarios para referir a los niños o padres que identifiquemos con situaciones particulares y condiciones especiales que no hayan sido atendidos. 
Tabla 2. Estrategia de gestión de las políticas públicas educativas

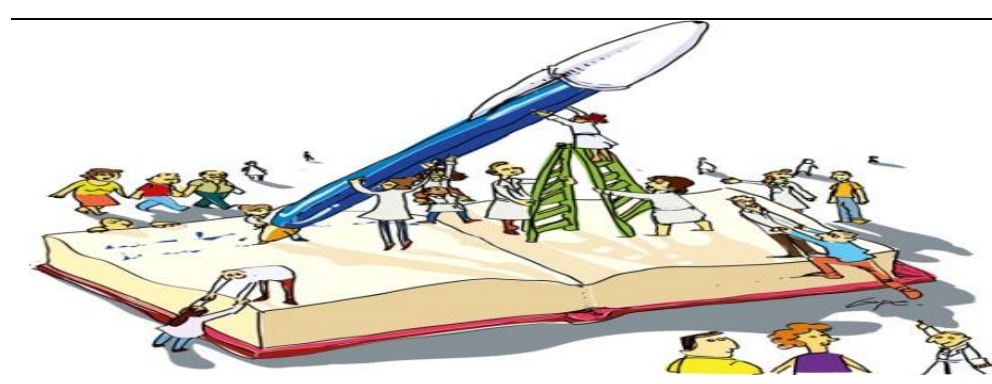

\section{Actividades}

1. Tomar decisiones en colectivo sobre los asuntos de la gestión escolar.

2. Resolver asuntos de interés de la comunidad educativa a través de sus vocerías en los comités.

\section{Dirigida: Al Consejo Educativo}

Objetivo: Promover la toma de decisiones, de carácter social democrático, responsable y corresponsable de la gestión de las políticas públicas educativas en articulación inter e intrainstitucional y con otras organizaciones sociales

- Gestionar acciones y soluciones ante entes gubernamentales.

- Establecimiento de alianzas. Esta tarea es considerada central para el cumplimiento de los objetivos que se proponen realizar. Las alianzas, son las formas más profundas de constitución de redes en donde se comparten recursos de modo estable, por períodos prolongados de tiempo, en función de objetivos y proyectos comunes.

Es necesario precisar con quienes se conformarán redes interescolares e intersectoriales. Explicitando los acuerdos de compromisos entre las partes intervinientes y la modalidad de funcionamiento que se establece. 
3. Desarrollar acciones conducentes al mantenimiento y conservación de la planta física.

4. Diseñar estrategias de desarrollo socioproductivo en consonancia con el P.E.I.C.

5. Es necesario identificar líderes actuales o potenciales en las familias y la comunidad, que puedan involucrarse en diversas actividades dentro del centro o escuela, para trabajar junto con los educadores de forma participativa, cooperativa e inclusiva.

6. Presentar trimestralmente ante la Asamblea Escolar el informe de los avances y los resultados de la gestión escolar.

7. Mantener permanente interacción con el entorno de la escuela.

Fuente: Elaboración propia. 
Cuadro 3. Estrategia de formación y preparación

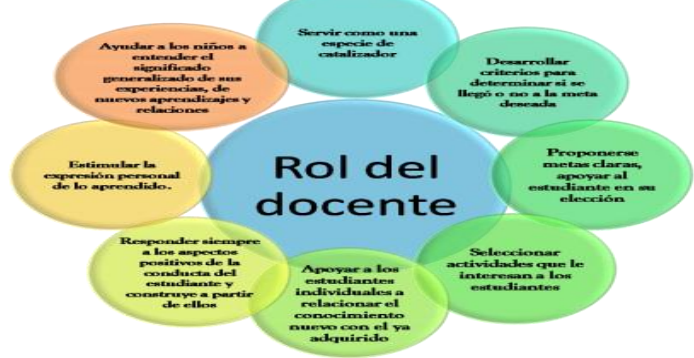

Actividades

1. Sensibilizar a los padres y miembros de la comunidad a fin de conformar brigadas de mantenimiento en el plantel.

2.Propiciar encuentros deportivos que fortalezcan la convivencia escolar, familiar y parroquial

3. Participar en actividades, junto con sus estudiantes, sobre conservación ecológica.
Dirigida: A los docentes en servicio

Objetivo: Promover el trabajo en equipo entre la escuela y los miembros de la comunidad.

Los docentes deben organizarse para la promoción del trabajo en equipo y para el establecimiento de alianzas entre el director y profesores para alentar el cambio.

- Promover que en conjunto entre la comunidad educativa y las organizaciones comunitarias del vecindario se llevan adelante iniciativas constructivas, positivas. Que deben considerarse en asamblea, contando con la autorización de la escuela para ser consideradas. Tales como:

- Una jornada voluntaria de arreglo y mantenimiento de la escuela.

- Una jornada para realizar un mural en una de las paredes públicas cercanas a la escuela y muy visibles para la comunidad.

- Una jornada de siembra de árboles, responsabilizando a familias o a salones. 
4. Propiciar la participación activa de los miembros de la Se deben planificar diversas actividades que logren acercar a todas las familias al comunidad con el fin de capacitar a los estudiantes en oficios centro o escuela y hacer que éstas se involucren, de alguna manera. Esto es menores (plomería, albañilería, carpintería) logrando su esencial para mantener relaciones recíprocas.

colaboración en el mantenimiento de los planteles.

Banco de recursos (voluntarios). Elaborar un documento que recoja información básica sobre los talentos y habilidades de los padres, que puedan beneficiar al centro o escuela. Además, se debe organizar el trabajo voluntario, proveer adiestramiento -de ser necesario- y reconocer el esfuerzo de cada participante. Esto aumentará la participación y la producción

Reuniones de padres del salón. En una reunión de padres, permita que cada familia se exprese, en una hoja de papel, sobre algunas preguntas que le permitan al maestro conocer las necesidades de su grupo (puede ser anónimo). Algunos ejemplos de preguntas podrían ser: ¿Qué espera de este centro o escuela con relación a la educación de su niño? ¿De qué manera le gustaría participar en el centro o escuela? ¿Qué preocupaciones tiene sobre la educación de su hijo?

Cuestionario de necesidades. Una de las formas de conocer a las familias es indagando cuáles son sus necesidades y tratar de suplirlas, con espíritu de reciprocidad, mediante recursos especializados en diversos temas o actividades. Se debe elaborar una lista con temas que el maestro entienda que podrían ayudar a los padres en su tarea de educar a sus hijos. Los padres también podrán sugerir algunos temas o actividades de su interés. Este cuestionario se entregará al inicio del año académico. 
5. Promover espacios para la formación de Escuelas con los padres, madres y/o representantes para orientar y solventar los problemas que afectan a la familia.

Fuente: Elaboración propia.
- La escuela que tenga como meta trabajar en conjunto con las familias deberá tomar en consideración que el concepto de familia ha ido cambiando con el tiempo. Conocer y aceptar esta realidad será una parte fundamental en las relaciones recíprocas entre la escuela y la familia. A raíz de esto, se crean dinámicas familiares más complejas, en las que, por ejemplo, ambos padres trabajan y la educación o cuido de los hijos se delega a extraños o a algún integrante de la familia extendida, compuesta de abuelos o tíos, entre otros.

- Es necesario entender las diferentes composiciones familiares que ocurren en la sociedad actual para poder trabajar con cada una según sus particularidades y necesidades. Entre éstas se encuentran la familia nuclear, la extendida, la que está compuesta de un solo padre o por parejas de un mismo sexo.

- Pueden solicitarse facilitadores de talleres, para dictarlos a las sociedades de representantes y a las organizaciones comunitarias, para compartir información y reflexionar en los vecindarios y en las escuelas 
Cuadro 4. Estrategia de participación social

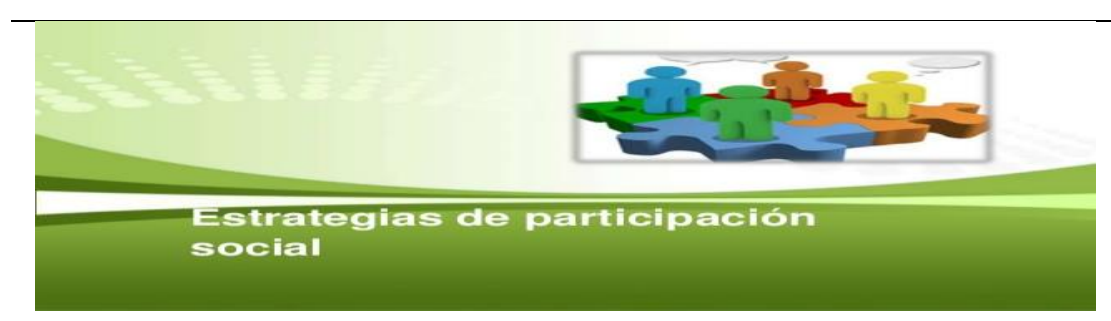

\section{Actividades}

1. Participar en jornadas de atención integral a los alumnos/as al comienzo del año escolar.

2. Participar en las reuniones de la conformación del consejo educativo al inicio del año escolar.

3. Participar como facilitadores en la capacitación de los estudiantes en oficios menores (plomería, albañilería, carpintería).

4. Participar en encuentros deportivos.

5. Participar como cronistas históricos de la comunidad.

6. Participar en actividades sobre cultura y conservación ecológica.

7. Participar en jornadas de atención integral a los alumnos/as al comienzo del año escolar.

8. Ofrecer sus servicios para reparar algún daño en la infraestructura de la escuela.

Fuente: Elaboración propia.
Dirigida: A los padres y representantes

Objetivo: Propiciar la participación de los padres y representantes como apoyo al docente y como estrategia para solventar necesidades institucionales.

- Involucrar a todos los actores, pero sincronizando sus roles y responsabilidades.

- Asegurar las condiciones y los mecanismos para hacer efectiva dicha participación 
Cuadro 5. Estrategia de participación estudiantil

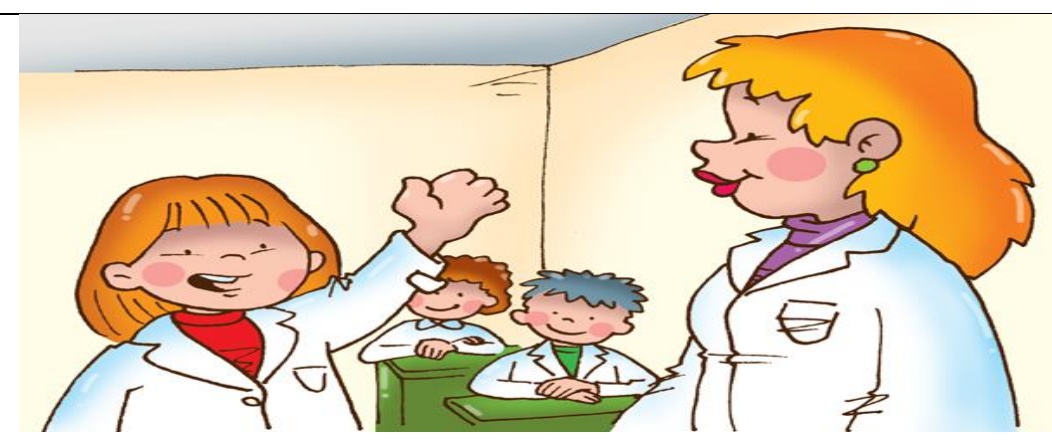

solución de problemas institucionales.

\section{Dirigida: A los estudiantes}

Objetivo: Participar de manera activa en proyectos institucionales y comunitarios

\section{Actividades}

3. Participar en actividades recreativas, deportivas, culturales y conservación ecológica.

2. Formar parte de las vocerías estudiantiles.

Fuente: Elaboración propia. 
Cuadro 6. Estrategia de servicios de apoyo a la institución

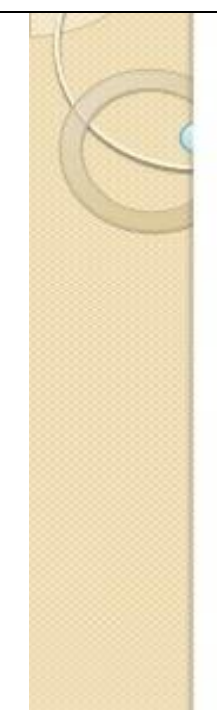

Dirigida: Al personal administrativo y obrero

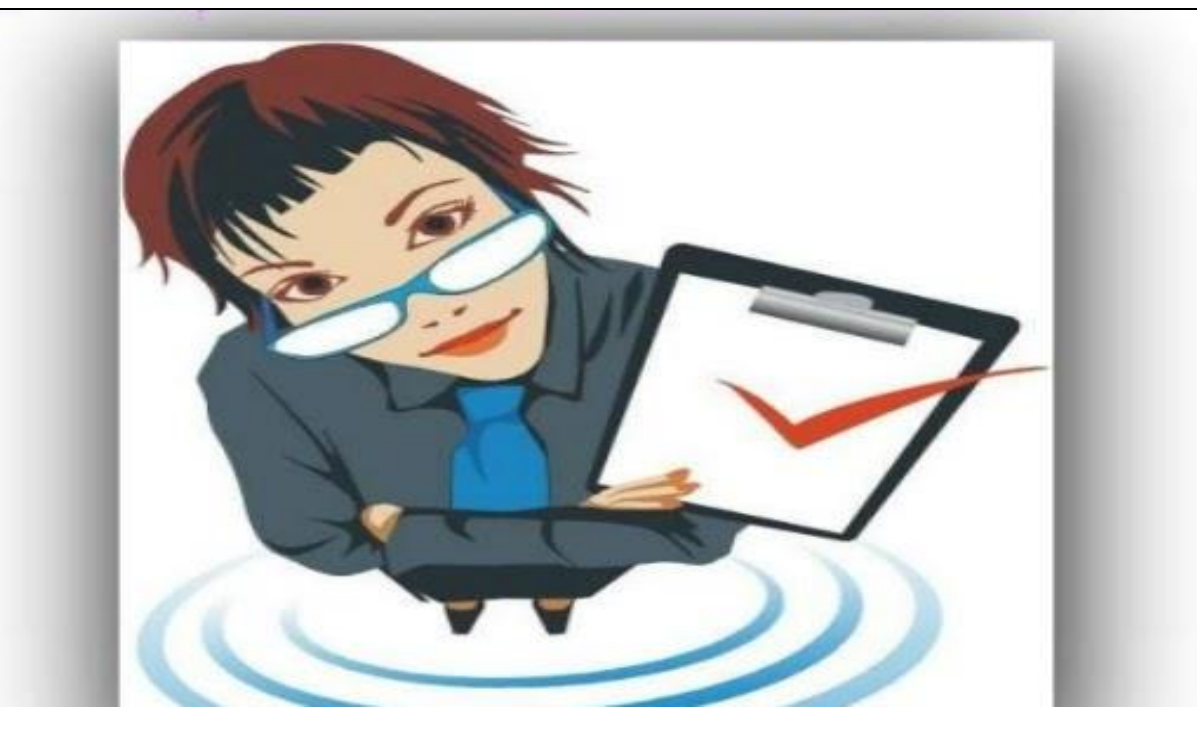

Objetivo: Apoyar la ejecución de proyectos comunitarios y jornadas institucionales en beneficio de la comunidad educativa.

Actividades

1. Participar en jornadas de formación de líderes comunitarios.

2. Participar en encuentros deportivos.

Fuente: Elaboración propia. 
CONCLUSIONES

En cuanto a los elementos teóricosfilosóficos y socioculturales contemplados en el diseño curricular del sistema educativo venezolano se observó que se orienta la concepción de la organización comunitaria como una participación protagónica que genera acciones educativas y sociales, cuyo fin es lograr soluciones sustantivas en las instituciones escolares. Para ello, es urgente y necesario promover espacios y experiencias comunitarias que atiendan las realidades en las cuales interactúan todos los actores involucrados en el ámbito escolar.

Con respecto a la descripción de la actitud de los actores que hacen vida en la Unidad Educativa Nacional Dr. Elías Rodríguez en relación con su participación en los procesos de gestión institucional se evidenció que existe una deficiente comunicación entre todos los entes que conforman la institución. Esto se fundamenta en la falta de trabajo en equipo en beneficio de la unidad educativa, por cuanto existe ausencia de un liderazgo institucional que promueva la participación comunitaria. Por otro lado, se observó un docente desmotivado y aislado donde solo se dedica al trabajo del aula y, por ende, padres y representantes ajenos totalmente a los problemas que aquejan a la comunidad estudiantil.

Finalmente, se diseñaron las estrategias dirigidas a la organización comunitaria de la institución educativa para promover la participación en los procesos de gestión institucional, las cuales constituyen un aporte considerable al problema planteado pues representan una guía de interacción directa con el entorno en el cual se encuentra la comunidad educativa. De allí la necesidad de que estas estrategias cumplan con su propósito socializador para que exista correspondencia con la realidad cultural de los estudiantes, del entorno familiar y del ámbito social.

\section{REFERENCIAS}

Aguilar M. y Ander-Egg, E. (2001). Diagnóstico social. Conceptos y metodología. Buenos Aires: Lumen Humanitas

Bisquerra, M. (2004). Metodología de la Investigación Educativa. Madrid: La Muralla

Constitución de la República Bolivariana de Venezuela. (1999). Gaceta Oficial. (30 de diciembre, $N^{\circ} 36.860$ )

Coronado, Y. (2010). Participación de los actores de la institución educativa en la gestión del cambio. El caso de una escuela pública de Lima. Proyecto desarrollado por la PUCP con apoyo del CONCYTEC

Crozier, M. y Friedberg, E. (1990). El actor y el sistema. Las restricciones de la acción colectiva. México: Alianza Editorial Mexicana

Freire, P. (2004). La Educación como Práctica de la Libertad. Argentina. Editorial Siglo XXI

Gómez, E. y Millán, L. (2002). La comunidad: espacio para la prevención. Caracas, Venezuela: Comisión Nacional contra el Uso llícito de la Droga (CONACUID)

González, H. (2007). Proyecto Comunitario: Una Gestión que se construye en conjunto. Mérida: Educere

Guánchez, L. (2014). Gerencia comunitaria. Universidad Nacional Experimental de las Fuerzas Armadas

Hernández S., R., Fernández C., C. y Baptista L., P. (2010). Metodología de 
la Investigación. (3ra. ed.). México: McGraw-Hill Interamericana

Ley Orgánica de los Consejos Comunales (2009). República Bolivariana de Venezuela Asamblea Nacional. Caracas, Venezuela. 4

Mengo, O. (2009). Investigación Documental. Universidad Central de Venezuela. Facultad de Agronomía. Cátedra: Introducción a la metodología de la Investigación Científica y Documental

Meza, M. (2011). El proyecto educativo integral comunitario: un instrumento para la promoción de la participación del docente. Revista Investigación y Postgrado, 26(1). Caracas: Venezuela

Ministerio de Educación, Ciencia y tecnología (s/f). El entorno educativo: la escuela y su comunidad. Programa Integral para la Igualdad Educativa (PIIE). Argentina

Ministerio de Educación, Cultura y Deportes. Viceministerio de Asuntos Educativos (1999). Proyecto Educativo Nacional. Caracas: Autor

Ministerio de Educación, Cultura y Deportes (2001). Bases Conceptuales y Operativas de las Escuelas Bolivarianas. Caracas, Autor

Ministerio del Poder Popular para la Educación (2007). Currículo Nacional Bolivariano: Diseño Curricular del Sistema Educativo Bolivariano. República Bolivariana de Venezuela. Caracas

Ministerio de Educación, Cultura y Deportes (2009). Ley Orgánica de Educación. Gaceta Oficial de la
República Bolivariana de Venezuela $N^{\circ}$ 12.428, Caracas-Venezuela

Ministerio del Poder Popular para la Educación (2012). Resolución 058, Consejos Educativos. Gaceta Oficial 397.068

Prieto Figueroa, L. B. (2006). El Estado Docente. Caracas: Fundación Biblioteca Ayacucho

Rodríguez, J. A. (2014). Aproximación a un modelo teórico de participación social que fortalezca la calidad de vida desde el contexto de la educación venezolana (tesis doctoral). Universidad de Córdoba

Sánchez, J. (1998). Métodos y Técnicas de Análisis de Contenido. Revista Internacional de Sociología, 43(1), 95 $-110$

Serrano L., A. K. (2018). Estrategias de organización comunitaria dirigida a los adultos para la transformación de los espacios educativos (trabajo de especialista). Universidad Nacional Experimental Simón Rodríguez (UNESR), Caracas, Venezuela

Universidad Nacional Experimental de las Fuerzas Armada [UNEFA] (2011). Organización social comunitaria. Recuperado de http://educacionparaeltrabajo8.blogspot .com/2011/01/organizacion-socialcomunitaria.htm

Vaagt, G. y Ruiz, J. (2008). Organización Comunitaria. Programa especial para la seguridad alimentaria nutricional nacional (PESANN). Instituto de Formación Permanente (INSFOP). Nicaragua 